\title{
Simulation of the Intake and Compression Strokes of a Motored 4-Valve SI Engine with a Finite Element Code
}

\author{
O. Bailly' , C. Buchou' ${ }^{1}$ A. Floch ${ }^{1 *}$ and L. Sainsaulieu' \\ 1 Technocentre Renault, Research Department, Sce 0073, 1 avenue du Golf, 78288 Guyancourt - France \\ * Engine Department \\ e-mail: christophe.buchou@renault.fr
}

\begin{abstract}
Résumé - Simulation de l'admission et de la compression dans un moteur 4-soupapes AC entraîné à l'aide d'un code de calcul à éléments finis - Un code de calcul utilisant une approche mixte éléments finisvolumes finis en tétraèdres a été développé pour les simulations moteur. Le code prend en compte le déplacement des parois mobiles comme les pistons et les soupapes de façon totalement automatique : un maillage unique est utilisé pour tout le calcul sans intervention de l'utilisateur. Un schéma implicite du quatrième ordre en espace et du premier ordre en temps est retenu. Le travail présenté dans cet article fait partie d'une démarche globale de validation de cette nouvelle approche pour les moteurs. Des comparaisons entre calculs et mesures lors des phases d'admission et de compression dans un moteur 4-soupapes AC y sont exposées.

Ces expériences sont conduites dans un moteur à accès optique et concernent l'intensité de la turbulence, les vitesses moyennes, le swirl et le tumble, toutes déduites de mesures par vélocimétrie laser. Nous y établissons des comparaisons à plusieurs endroits et instants du cycle, ainsi que sur des quantités globales (swirl et tumble). L'accord constaté entre simulations et mesures est excellent.
\end{abstract}

Mots-clés : simulation, éléments finis, moteurs.

Abstract - Simulation of the Intake and Compression Strokes of a Motored 4-Valve SI Engine with a Finite Element Code - A CFD code, using a mixed finite volumes-finite elements method on tetraedrons, is now available for engine simulations. The code takes into account the displacement of moving walls such as piston and valves in a full automatic way: a single mesh is used for a full computation and no intervention of the user is necessary. A fourth order implicit spatial scheme and a first order implicit temporal scheme are used. The work presented in this paper is part of a larger program for the validation of this new numerical tool for engine applications. Here, comparisons between computation and experiments of the intake and compression strokes of a four-valve engine were carried out.

The experimental investigations are conducted on a single cylinder four valve optical research engine. The turbulence intensity, mean velocity components, tumble and swirl ratios in the combustion chamber are deduced from the LDV measurements. The comparisons between computations and experiments are made on the mean velocity flow field at different locations inside the chamber and for different crank angles. We also present some global comparisons (swirl and tumble ratios). The simulation shows excellent agreement between computations and experiments.

Keywords: simulation (CFD), finite elements, engines. 


\section{INTRODUCTION}

Cleaner emission and improved fuel consumption are main environmental issues for automotive manufacturers. To achieve these goals at a low cost, engine emissions must be improved. The mixture preparation is thus of primary importance and the use of an efficient numerical tool is of major interest for the engine designers, to help them understand the flow structure inside the combustion chamber.

One way to achieve stable combustion of lean mixtures is to generate a swirl motion during the induction process [1]. In current multi-valve engines, swirl motion is generated by a port deactivation [2] and its magnitude is controlled by intake port and combustion chamber geometries. But we know that this swirl motion is sensitive to the port excentration and one often observe a tilted charge motion [1,3] in four-valve pentroof engines. Such geometries thus offer a very good test case for a numerical tool such as N3S-Natur. Furthermore, it is expected that the use of this tool will help optimize the chamber and port geometries.

The first difficulty encountered when doing numerical simulations if to build a mesh and to control the mesh deformation. N3S-Natur offers an elengent solution to this problem since a single mesh is needed for a full computation and the mesh movement is handled is a full automatic way. The second difficulty is to check the accuracy of the computations.

This problem is now controlled and it is current to compute intake and compression strokes. The second problem is to ensure that the code gives accurate results. It is for this reason that comparisons between numerical and experimental results must be made $[5,6]$. When the numerical results are reliable enough, it is possible to compute different intake designs and choose the best $[4,7]$.

The paper is organized as follows. First, the CFD code, the LDV system and the engine configuration are described. The in-cylinder swirl motion induced by a port deactivation during intake and compression strokes is then analyzed. Global comparisons (swirl and tumble ratios) and local comparisons (mean velocity components in several locations) are made in several sections. Finally, computed and measured turbulence intensities near the spark plug are compared and discussed.

\section{THE CFD CODE}

Usually, CFD codes used to compute intake and compression strokes, show several severe limitations: the most frequent weaknesses of these codes are the mesh motion, the robustness of the numerical methods and the validity of the physical models. Several months are usually needed to perform a single computaion. This jumbo delay prevents the use of such numerical tools for the purpose of engine design.
In order to overcome the problems related to stability and robustness, a new code, based on modern numerical methods, called N3S-Natur, has been developped by several partners. This code uses a mixed finite volumes (for convective terms) - finite elements (for diffusive and source terms) method based on tetraedrons. The numerical approximation of the convection terms is obtained by using Roe's linearization [10]. Quasi fourth order accuracy in space is achieved thanks to a Muscl technique $[8,9$,$] . On the other$ hand, the code allows moving walls such as piston and valves. The mesh motion handling is very robust, even when the mesh is extremely distorded such as in valve curtains (valve opening and valve closing) or at TDC.

The equations solved by the code are the Navier-Stokes equation with a $k-\varepsilon$ turbulence model with compressibility terms [11]. A standard law of the wall is used for the description the velocity and temperature profiles near walls. The dynamic viscosity of the flow mixture is estimated by using a Sutherland law.

\section{MESH GENERATION}

The mesh of this engine was generated from the CAD files by using Ansa (for the mesh of the surface) and GHS3D (for the volumic mesh). A 3D view of the geometry is showed in Figure 1. Three weeks were necessary to perform the full mesh generation. The mesh is composed of 113000 nodes, 605000 cells and 52000 wall faces. The mean volume of the elements in different parts of the geometry is given in Table 1.

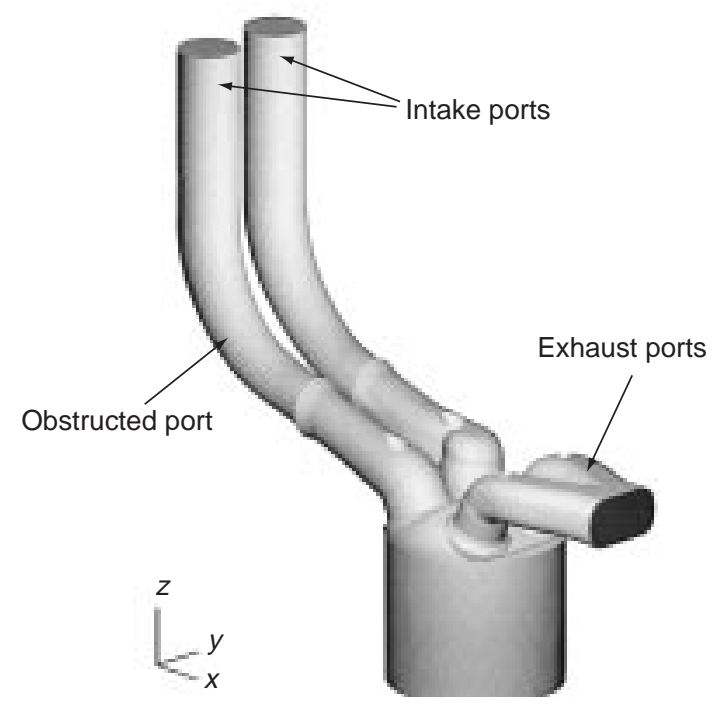

Figure 1

$3 \mathrm{D}$ view of the geometry. 
TABLE 1

Mean volume of the elements

\begin{tabular}{l|c|c}
\hline & Chamber & Intake ports \\
\hline Mean volume at TDC & $0.18 \mathrm{~mm}^{3}$ & $1.2 \mathrm{~mm}^{3}$ \\
Mean volume at BDC & $6.06 \mathrm{~mm}^{3}$ & $1.2 \mathrm{~mm}^{3}$ \\
\hline
\end{tabular}

\section{ENGINE SIMULATION}

The engine is a research four-valve-per-cylinder sparkignited configuration. This engine has a pent roof combustion chamber with central spark plug, separated intake ports and a flat top piston. Some of the engine specifications are summarized in Table 2. Computations are carried out with intake port deactivated.

TABLE 2

Specifications of the engine

\begin{tabular}{l|l}
\hline Bore & $80 \mathrm{~mm}$ \\
Inlet port & Helical + plain directed \\
Compression ratio & 9.5 \\
Combustion chamber & Pent roof \\
Valve per cylinder & 4 \\
Engine speed & $2000 \mathrm{tr} / \mathrm{min}$ \\
Intake valve lift & $8.035 \mathrm{~mm}$ \\
Intake valve opening & $-9 \mathrm{CAD}$ \\
Intake valve closing & $19 \mathrm{CAD}$ \\
\hline
\end{tabular}

The computation runs from the intake TDC (-360 CAD) to $30 \mathrm{CAD}$ after the compression TDC. The time step is $8.310^{-6}$.s (one time step corresponds to $0.1 \mathrm{CAD}$ ). In the whole computational domain, we considered an inert gaz with properties equivalent to those of air.

The initial conditions are given in Figure 2. The initial velocity is set to zero and the initial temperature and pressure are uniform in both the chamber and the intake ports. Nevertheless we used different values in the chamber and the ports since these conditions are determined by performing a separate simulation with the 1D simulation tool Vectis and it was not possible to reproduce the gradients of the scalar fields.

The turbulent boundary conditions at walls are determined by a standard wall law. The intake conditions (velocity, pression) are determined by measurements. The intake temperature is constant and equal to $300 \mathrm{~K}$.

The CPU time of this computation was 380 hours on a Cray C94, at the time it was performed. This very large CPU time originates in two reasons: first, the numerical method

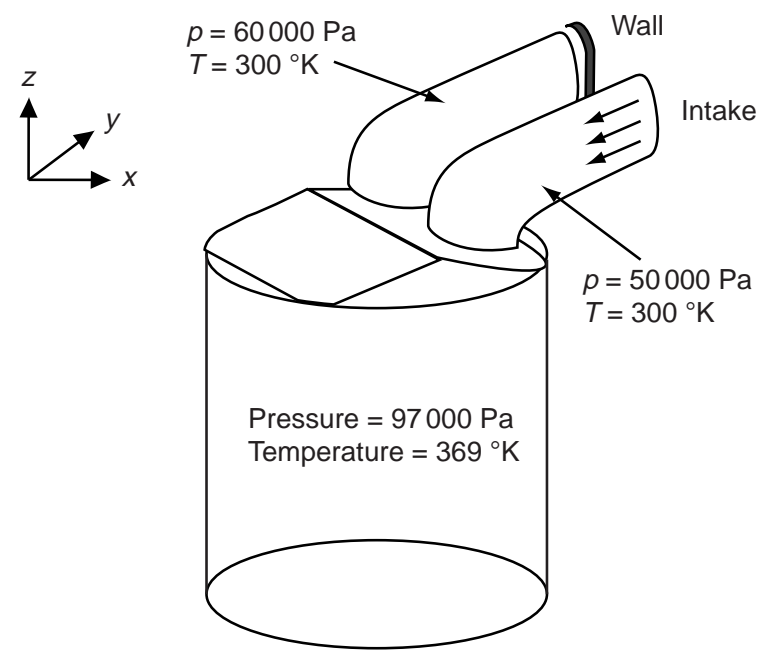

Figure 2

Initial conditions.

used for the solution of the sparse linear system, involved by the implicit numerical method, is poorly vectorised. During this phase, which is the most time consuming one, the Cray is used as a scalar computer. Obviously, the numerical methods of N3S-Natur are better suited for parallel computing. Second, no remeshing was available at the time of this computation. At the end of the compression stroke, the cell number could be reduced by a remeshing step. The maximum memory used is equal to $79 \mathrm{Mw}(632 \mathrm{Mo})$.

\section{THE EXPERIMENTS}

\subsection{The Engine}

The engine used for this study is a single cylinder engine equipped with a multicylinder cylinder head. The cylinder and piston are extended from the original ones. The first cylinder presents several optical accesses on the cylinder liner and in the cylinder head. A fixed mirror inclined at 45 degrees is inserted in the extended piston. The engine was motored for all tests.

\subsection{The LDV Experiments}

Velocities have been measured with a laser doppler velocimeter (LDV) in the cylinder and in the pent roof combustion chamber, in back scatter light diffusion configuration. The LDV set-up is a Dantec 2-components; the laser source is a $5 \mathrm{~W}$ argon-ion laser operating at 488 and $514 \mathrm{~mm}$. Two 
beams $38 \mathrm{~mm}$ apart are focused with $310 \mathrm{~mm}$ focal lens. The probe volume size is about $0.09 \mathrm{~mm}$ wide and $1.5 \mathrm{~mm}$ long. Instantaneous velocity signals were obtained and processed by Dantec BSA.

Figure 3 shows the location of the LDV measurements in the cylinder liner and in the combustion chamber. Nine points are located along diameters parallel and perpendicular to the pent roof edge, every $9 \mathrm{~mm}$, in three sections located $20 \mathrm{~mm}, 40 \mathrm{~mm}$ and $60 \mathrm{~mm}$ from the cylinder head gasket section. The tangential and axial velocity components were measured in each point. Ten points are located along the cylinder axis. Tangential velocity components are measured along this axis. The LDV measurement locations are selected to compute the evolution of the angular momentum of the swirl induced motion, during the intake and compression strokes.

The mean motion and turbulence characteristics at and around the spark plug gap location are of particular relevance for the initiation of combustion and flame kernel growth. Velocity measurements have thus been done in three points along an axis $8 \mathrm{~mm}$ below the pent roof edge. The middle point is located under the spark plug. The two other ones are located $20 \mathrm{~mm}$ from the central point, on both sides. In each location, the tangential velocity component has been measured. More details on the experimental set-up are given in [12].

\section{RESULTS}

\subsection{Computational Results}

Figure 4 shows computed the flow structure, at the earlier stage, during induction (300 CAD BTDC), on the cutting planes A-A, B-B and C-C, corresponding to the regions located respectively under the helical port, the spark plug and the deactivated port. Flow pattern is also given in the plane D-D, parallel to the apex. At the beginning of the intake stroke, the inlet flow through the valve curtain area sets up a ring-shaped vortex located under the helical inlet valve. Then, the $2 \mathrm{D}$ velocity field become $3 \mathrm{D}$ fields as two vortices appear (see sections $\mathrm{AA}, \mathrm{BB}$ and $\mathrm{DD})$.

At the middle stage of the inlet stroke (270 CAD BTDC), as the valve lift reaches high values, the flow in the port is guided to the upper part of the curtain area (see Fig. 5). The vortex located in the upper region moves towards the exhaust side of the engine, thus filling the whole in-cylinder volume. The secondary vortex formed under the inlet valve and along the cylinder wall is weaker.

A large-scale well-defined swirl structure (single cylinderfilling vortex), appears near BTDC (Fig. 8a). Note that in each section, the center of rotation does not coincide with the cylinder axis. This indicates that the axis of the swirl vortex is tilted form the cylinder axis. Furthermore, the rotation axis

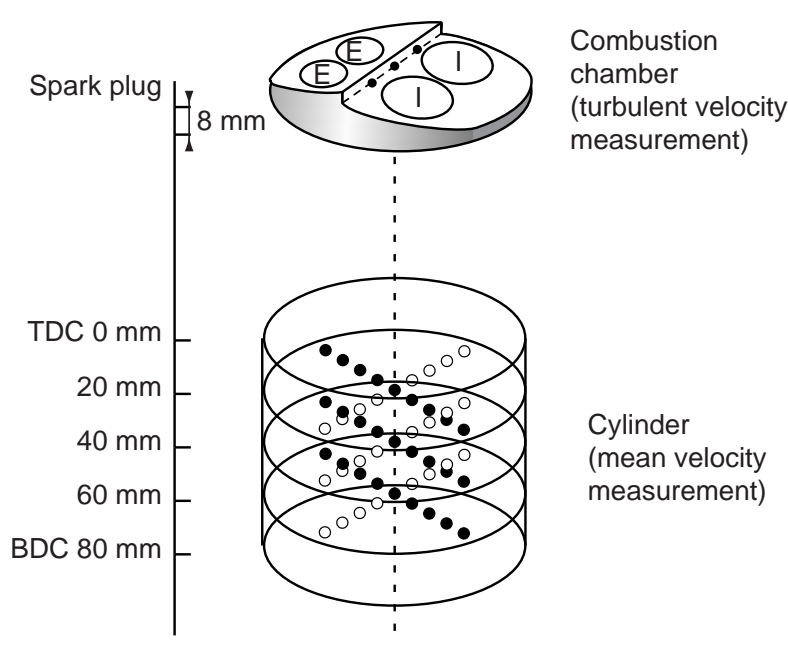

Figure 3

LDV measurement locations in the chamber.
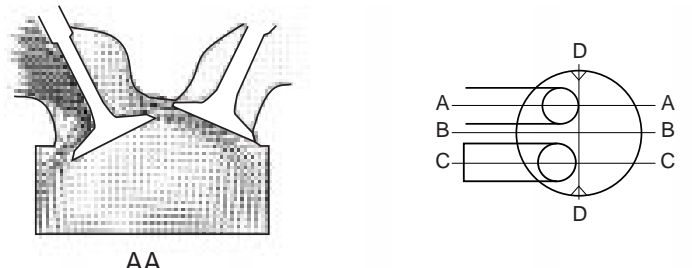

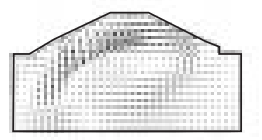

$\mathrm{BB}$

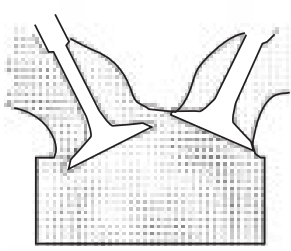

$\mathrm{CC}$

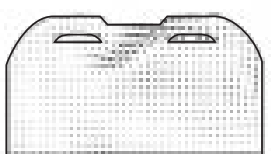

DD
Crank $=-300 \mathrm{CAD}$

Figure 4

Vertical velocity fields at $-300 \mathrm{CAD}$. 
is inclined simultaneously, from the exhaust side to the inlet one and from the helical port to the deactivated port.

In Figure 6, the corresponding vertical velocity fields in the sections perpendicular and parallel to the apex are shown. We notice a strong vortex, namely the tumble and crosstumble components of the charge motion.

At IVC (Fig. 7b), the swirl motion is well established in the whole cylinder volume, even in the combustion chamber. The swirl axis has described a 90 degrees rotation around the cylinder axis. The center of the rotation is located under the inlet valves in the upper region of the cylinder, and under the exhaust valves in the lower part. As the piston moves upward, the rotation axis of the swirl vortex tilts more and more.

Computed values of SR (swirl ratio), TR (tumble ratio) and CR (cross-tumble ratio) are given in Figure 8 (the formula used to compute these ratios are given in the Appendix). Since a large-scale well-defined swirl structure appears around 270 CAD BTDC and since measurements are not available at the end of the compression strokes, the comparison in Figure 8 is restricted to crank angle between $270 \mathrm{CAD}$ and $60 \mathrm{CAD}$. According to computation, the swirl ratio reaches a maximum during the intake stroke and then decreases monotonically during the compression. During the end of the intake stroke, the tumble ratio decreases to reach a moderated value at IVC (less than 1), while cross-tumble ratio increases to reach a constant value at the same time. These results indicate as previously that the axis of the swirl
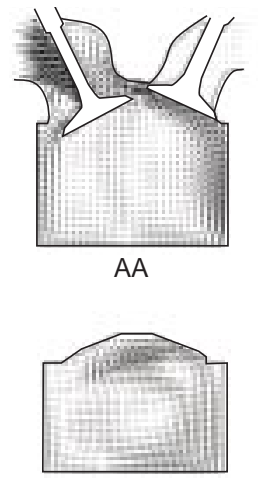

BB

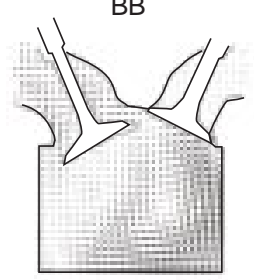

CC

Figure 5

Vertical velocity fields at -270 CAD.

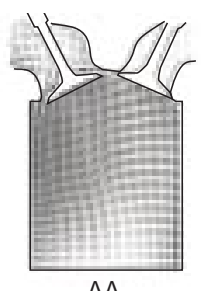

AA

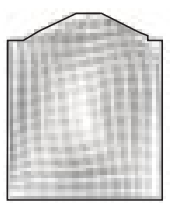

BB

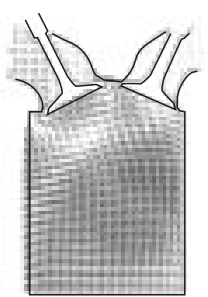

$\mathrm{CC}$

Figure 6

Vertical velocity fields at $-180 \mathrm{CAD}$.
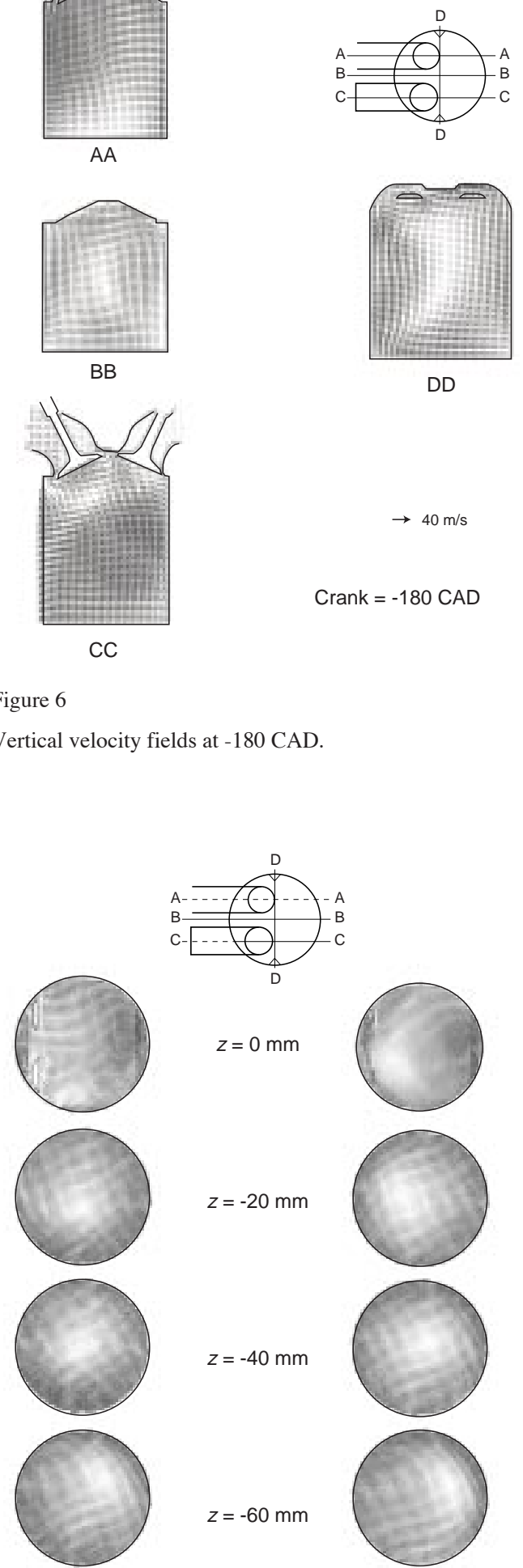

DD

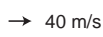

Crank $=-180 \mathrm{CAD}$ 
motion is inclined at the end of the intake stroke. After the inlet valve closing, the swirl axis moves and is mainly tilted in a vertical section cutting the apex.

\subsection{Comparison Between Experimental And Computational Results}

The comparison of the computed and measured values of SR, TR and CR are given in Figure 8. The swirl ratio curves show a very good agreement. Concerning the tumble ratio, the behaviours are nearly the same, but small differences are noticed, in particular during the intake stroke. These discrepencies may originate in two causes: the measurement points used for the computation of the tumble ratio are not in a sufficient number and the initial conditions (null velocity) imposed at the begining of the computation are not correct.

Velocity flow fields, on the vertical cross sectional planes A-A, B-B, C-C and D-D as shown in Figure 4 and on the horizontal cross sectional planes at different $z$ locations from the cylinder head gasket section $(0 \mathrm{~mm}, 20 \mathrm{~mm}, 40 \mathrm{~mm}$ and
$60 \mathrm{~mm}$ ), were extracted from the computed flow fields and plotted together with the experimental datas, at $150 \mathrm{CAD}$ BTDC, as shown on Figures 9 and 10. The agreement between computation and experiments is excellent. This observation confirms the swirl ratio and tumble ratio comparisons (Fig. 8).

The prediction of the axial velocity profiles is very good but not as excellent as the prediction of the tangential velocity profiles. We suspect that the law of the wall we used is insufficient and that compressibility terms should be used.

We conclude this section with a comparison of the measured and computed turbulence intensities: the measured intensity is deduced from the instantaneous flow field by ensemble averaging [1]. The computed one is given by the $k-\varepsilon$ model. The results shown in Figure 11 correspond to a spatial average over the three points just below the spark plug. We see on Figure 11 that the numerical predictions are very good. At the end of the compression stroke, some differences may be observed but we assumed that the turbulence is isotropic which is obviously false at that time.
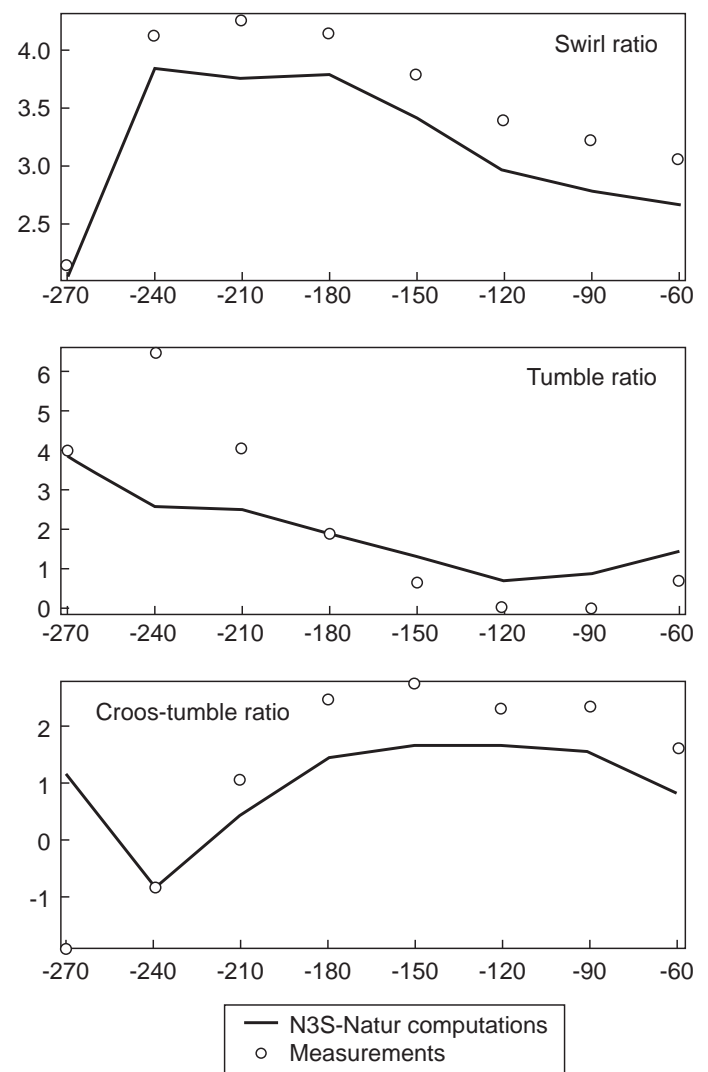

Figure 8

Comparison about the swirl, tumble and cross-tumble ratios.
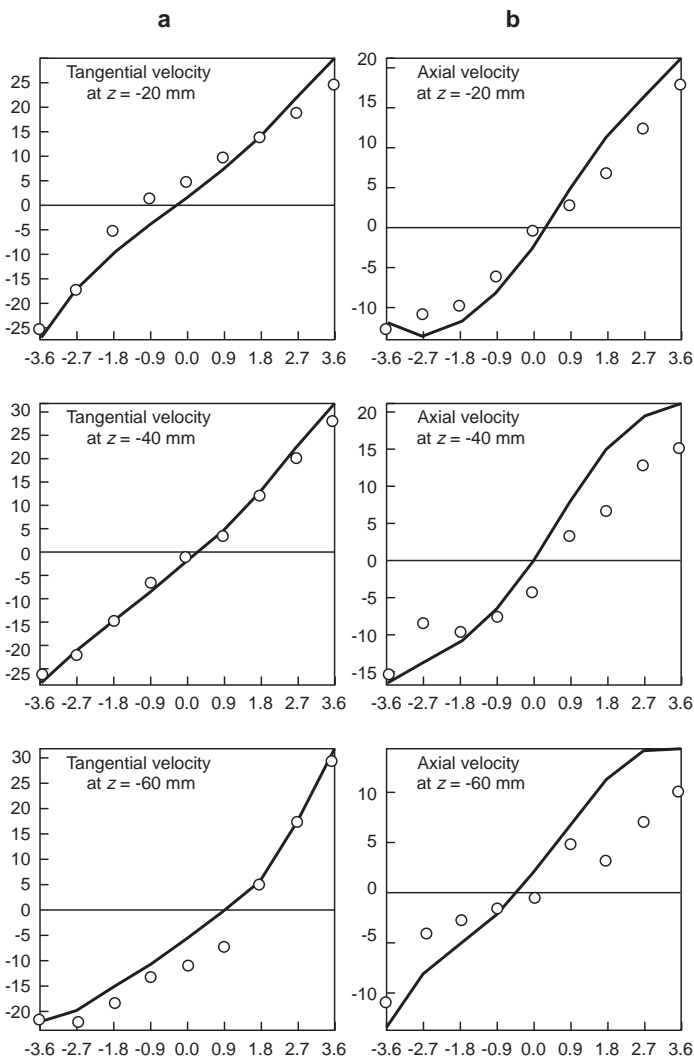

- N3S-Natur computations Measurements

Figure 9

Velocities at $-150 \mathrm{CAD}(\mathrm{m} / \mathrm{s})$. Parallel plane to the pent roof edge $(x=0)$. 
a
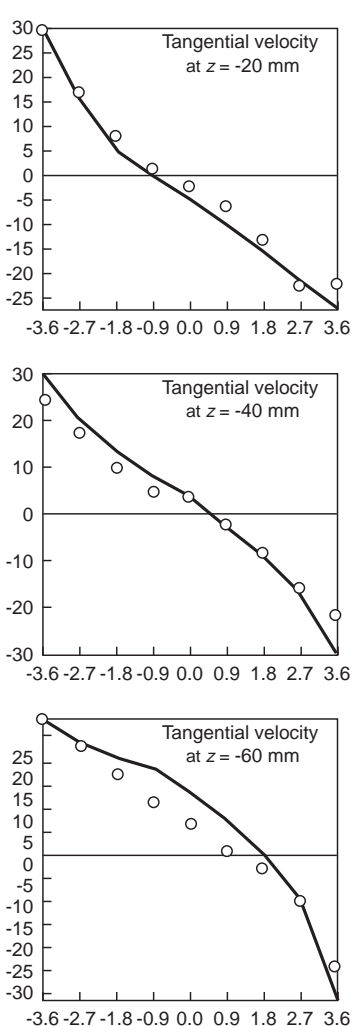

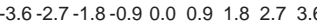

\section{- N3S-Natur computations}

- Measurements

Figure 10

Velocities at $-150 \mathrm{CAD}(\mathrm{m} / \mathrm{s})$. Perpendicular plane to the pent roof edge $(y=0)$.

b
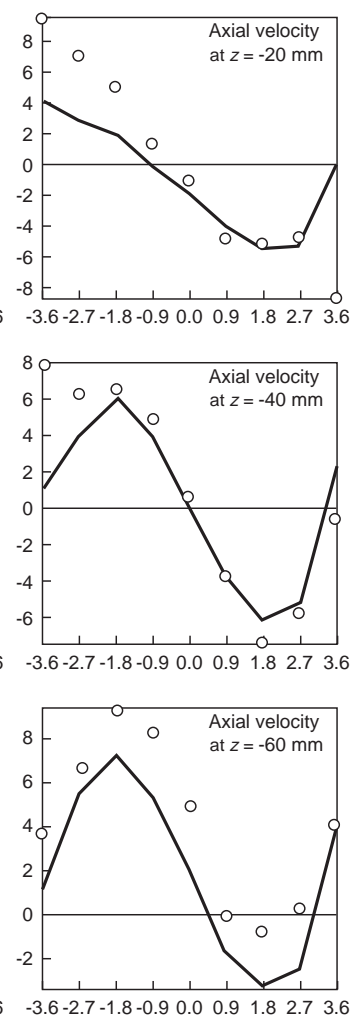

(1)

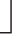


8 Van Leer, B. (1972) Towards the Ultimate Conservative Difference Scheme: the Quest of Monotonicy. Lecture Notes in Physics, 18, 163.

9 Carpentier, R. (1995) Approximation et analyse numérique d'écoulements instationnaires. Application $\$$ des instabilités tourbillonnaires. PhD Thesis, University of Nice.

10 Roe, P.L. (1981) Approximate Riemann Solvers, Parameters Vectors and Difference Schemes. J. Comp. Phys., 43, 357.

11 Morel, T. and Mansour, N.N. (1982) Modeling of Turbulence in Internal Combustion Engines. SAE 820040.

12 Floch, A. and Baby, X. (1997) Investigation of the Incylinder Tumble Motion in a Multi-Valve Engine: Effect of the Piston Shape. SAE 971643.

\section{APPENDIX}

The swirl and tumble ratios are given by:

$$
\text { Swirl }=\frac{\int_{v} r^{2} V_{\theta} \mathrm{d} r \mathrm{~d} \theta d z}{2 \pi \omega \frac{R_{\text {mes }}^{4}}{4}\left(Z_{\text {max }}-Z_{\text {min }}\right)}
$$

where:

$\omega \quad 2 \pi \mathrm{N} / 60$ ( $N$ is the speed engine in $\mathrm{tr} / \mathrm{min}$ );

$R_{\text {mes }} \quad$ is the measurement radius;

$z_{\max }-z_{\min }$ is the distance between the upper and the lower measurement planes.

The tumble ratio is calculated on a plane located at the middle between the piston and the cylinder head edge.

$$
\text { Tumble }=\frac{\int_{0}^{2 \pi} \int_{0}^{R_{\text {mes }}} r^{2} V_{\theta} \mathrm{d} r \mathrm{~d} \theta}{2 \pi \omega \frac{R_{\text {mes }}^{4}}{4}}
$$

The integrals are calculated by using a first order approximation. 